Contact stresses in gelatin spheres under compressive die loading

Andrew J Rosenthal

Andrew.Rosenthal@nottingham.ac.uk

Division of Food Science

School of Biosciences

University of Nottingham

Sutton Bonington Campus

Sutton Bonington

Loughborough

LE12 5RD

\title{
Keywords
}

Compression testing

Fruit

Gel testing

Photoelasticity

Puncture test

Stress loading

Vegetable

\section{Practical applications}

The common practice of supporting materials on a plane surface during texture testing is brought into question due to differences in contact stress when supported in this manner as opposed contouring the object in some kind of supporting mould.

Soft gels which deform under gravity maybe tested with a texturometer if immersed in a liquid that provides buoyancy. 


\section{Abstract}

Gelatin spheres have been used to model stress/strain behaviour of spherical foods (e.g. fruits) during compression testing. Photoelasticity reveals differences in stress distribution within gelatin spheres depending on the way in which the spheres are supported. Moreover, these methods of support appear to result in differences in the elastic modulus at higher levels of applied stress.

If differences in the manner of support of model foods under compression result in variation in the stress distribution and moduli, then thought must be given to the way in which real foods are supported during quality and texture testing.

\section{Introduction}

Kilcast, Boyar and Hudson (1984) introduced gelatin photoelasticity as a technique for measuring stress distributions in food gels during penetration testing. However, as a technique photoelasticity has not been embraced by the food science community. This is despite the fact that gelatin has been reported as being one of the most photoelastic materials available for the study of stress. Moreover, as a material, gelatin is easily moulded and modelled. This lack of take up is perhaps due to the fact that gelatin has a relatively low modulus which means that birefringent isoclines can develop merely through deformation due to its own mass under gravity (Kuske and Robertson, 1974) which is a disadvantage in many stress analysis situations. Furthermore as a modelling material it has the further disadvantage in that it tends to dry out and and shrink during lengthy experiments. Generally stiffer and more stable materials such as epoxy resin, polymethacrylate, or polycarbonate have been used to model structures. In some respects, advances in computational methods of finite element stress analysis have replaced photoelasticity as a technique in the study of stress distributions within engineering materials.

The literature on food quality and texture testing contains references to many compressive tests, whether using simple hand held puncture testers, multiple headed tenderometers, two bite imitative protocols (e.g. Texture Profile Analysis), established industry standards (e.g. Bloom gelometer), to mention but a few (see for example Kramer and Twigg, 1970, Bourne, 2002, Rosenthal, 2015). 
Many fruits and vegetables are roughly spherical in shape and when low strains are applied in compression, the stresses develop through two contact regimes

(Figure 1). Plate loading employs a flat plate larger than the area in contact with the food and die loading in which the point of contact is smaller than the curvature of the sample. In the case of plate loading the stress is greatest at the centre where the curvature is most deformed by the plane contact surface, and the stresses tend to zero as the curvature of the food loses contact with the plate. In contrast the stresses in die loading rise parabolicly from the centre towards the perimeter of the die. The stresses at the centre being due to elastic deformation while shear stresses progressively increase along the radius towards the perimeter.

A common way of undertaking compression tests on spherical (or roughly spherical) foods is to place the sample on a base plate and then to bring the test geometry (die or plate) into contact with the food. Bizarrely, we tend to focus our attention on the test geometry being brought into contact with the sample, while relatively little consideration is taken of the contact stresses between the base plate and the food. This research note raises questions pertaining to how samples are supported during compressive testing of roughly spherical foods.

\section{Procedure}

Two hundred and forty Bloom Pig Skin gelatin (MM Ingredients, Wimborne, UK) was suspended in deionized water at a concentration of $6 \%(\mathrm{w} / \mathrm{w})$. The mixture was warmed on a magnetic stirrer until fully dissolved. Two part silicone rubber ice moulds (Dunelm Mill, Leicester, UK) were wiped with a paper towel which had been dipped in a light mineral oil (WD40, San Diego, USA) to act as a mould release agent. The dissolved gelatin solution was then poured into the rubber mould. The solution was degassed by applying 400 mBar vacuum to the mould for one minute the mould was then refilled and subjected to $100 \mathrm{mBar}$ vacuum for 20 seconds. Finally the mould was topped up with further gelatin solution. Moulds were placed in a refrigerator at $4{ }^{\circ} \mathrm{C}$ overnight. To remove the gelatin sphere, the mould was immersed in iced water and the edge of the mould top was gently separated from its base. While keeping immersed in ice water the two halves were opened to release the gelatin sphere. The mould filling hole left an irregularity on the surface of the 
sphere, though care was taken during subsequent testing to avoid contact between this irregularity and the contact surfaces. Once removed from their mould, gelatin spheres were retained in ice water until ready for use.

A CT3 Texturometer (Brookfield, Harlow, UK) was fitted with a $10 \mathrm{~mm}$ diameter black plastic cylindrical probe. A glass sided tank was placed on the base plate to accommodate the samples. Testing was undertaken with the tank full of iced water. Samples were either:

- Placed on the base plate (a $1 \mathrm{~mm}$ thick aluminium ring was positioned at the centre of the tank to prevent the sphere from rolling)

- Held in a cut-away rubber mould - identical to those used to form the spheres, but with the front and back removed to allow the passage of light. Illumination was from a Sodium lamp with a polarizing filter. A Nikon D40 camera with a polarizing filter was arranged on the other side of the tank. The camera was set to manual focus, aperture priority, F25 and with a digital equivalent of ISO 1600 film speed. Prior to placing the sample in the tank, the polarizing filter on the camera was aligned to achieve cross polarized illumination relative to the filter on the light source.

The test procedure involved moving the probe to the surface of the sphere until a trigger of $67 \mathrm{mN}$ was achieved, the probe was driven at $0.1 \mathrm{~mm} \mathrm{~s}^{-1}$ to a target stress of $300 \mathrm{mN}$. Once the target stress was achieved, the deformation was maintained for 60 seconds during which time photographic images of the sphere under cross polarised light were collected. Following the initial compression, the probe was raised and the procedure repeated but to a new target stress $300 \mathrm{mN}$ greater than the last applied stress. This process was continued, each time increasing the stress by a further $300 \mathrm{mN}$ until the sphere ruptured.

Triplicate experiments were undertaken for each support type (i.e. three spheres per support). While the texturometer was set to apply a particular force, the actual force was divided by the contact area of the probe, yielding a contact stress. A buoyancy correction was calculated from the dimensions of the probe, the depth of probe penetration, the height of the upper surface of the sphere from the base of the tank (being higher with the mould support compared to the plate alone), and the depth of 
the water in the tank. The strain was estimated from the depth of deformation of the sphere in relation to its original dimensions.

\section{Results and discussion}

The methodology used in this study evolved through a series of trials. Initially the procedure undertaken by Kilcast, Boyar and Hudson (1984) was followed, however unlike their rectangular block, the sphere used in this study focused the light through refraction to the centre. On the basis that the refractive index of the gelatin gel was similar to that of water and light passing perpendicular through a plane glass surface would not refract, a water filled glass tank was adopted. This tank had the further advantages that it provided buoyancy which supported the sphere from gravitational distortion and it prevented the gelatin from drying out. Figure 2 shows the images of spheres supported on both a plate and a mould. There are clear differences in the distribution of the isoclines, the plate support having predominantly vertical parallel stresses suggesting they are being exerted by contact of both the probe and the base plate. While the mould supported sphere shows the isoclines that radiate in an oyster shell pattern from the probe tip. The supporting mould appears to cushion and spread the stresses which generally take on the shape of the mould forming contours towards the probe.

Figure 3 is an X-Y scatter plot of strain against buoyancy corrected contact stress. Within the data for each support regime, the variation is greatest in the strain component. This could be due to poor positioning of the sphere in relation to the probe, thus triggering the texurometer when only partial contact occurred. Despite the variation in the replicates there are clear differences between the plate and mould supported spheres. At low levels of stress and strain the data overlap, but as the applied stress increases the corresponding strains diverge. The slope of the best fit line is the reciprocal of the elastic modulus and for the plate supported spheres this modulus is equal to $146 \mathrm{kNm}^{-2}$ compared to $201 \mathrm{kNm}^{-2}$ for those contained in a mould. This substantial difference in moduli reflects the restraining nature of the mould which in addition to spreading the contact stresses also limits lateral deformation. Of course $6 \%$ gelatin is a relatively soft material and one which will 
rupture at relatively low stresses. Yet these spheres are not a bad model for fruits which soften as they ripen and in the case of berries are of a similar consistency. Data presented in figure 3 is limited to stresses of $35 \mathrm{kNm}^{-2}$ because at higher stresses some of the spheres ruptured. As might be expected the higher strains exhibited by the plate supported spheres, generally caused them to rupture at lower stresses than the mould supported ones.

It could be argued that the parallel between these model spheres and fresh fruit/vegetables is tenuous, for while the supporting mould employed here fits the shape of the sphere perfectly, providing a similar mould for each item of fresh produce is impracticable. Yet Bourne and Mondy (1967) achieved a similar effect by supporting potatoes in a bed of sand which presumably distributed the supporting stresses. This mode of support moulds itself to the outline of each item of produce and if used on a gel like material or a soft fruit, would likely provide containment akin to the rubber mould used in this study. In addition to better spreading the supporting stresses of an irregularly shaped fruit, the measured modulus would likely appear firmer and the lateral restriction to deformation may well limit barrelling exhibited by some soft unlubricated materials.

Sadly there are technical difficulties that prevent using this photoelastic technique with gelatin spheres embedded in sand. However, supporting food samples in sand should prevent localised support stress concentration and compression tests of such samples may provide a truer measure of modulus as well as higher rupture strengths.

\section{Ethical statement}

The author declares that he has no conflict of interest.

This study did not involve any human or animal testing.

\section{Acknowledgement}

I am grateful to John Mitchell and Malcolm Bourne for invaluable suggestions and encouragement.

\section{References}

BOURNE, M. C. 2002. Food Texture and Viscosity: concept and measurement, Geneva, New York, Academic Press. 
BOURNE, M. C. \& MONDY, N. 1967. Measurement of whole potato firmness with a universal testing machine. Food Technology, 21, 97-100.

KILCAST, D., BOYAR, M. M. \& HUDSON, J. B. 1984. Gelatin Photoelasticity: A New Technique for Measuring Stress Distributions in Gels During Penetration Testing. Journal of Food Science, 49, 654-655.

KRAMER, A. \& TWIGG, B. A. 1970. Quality Control for the Food Industry, Westport Connecticut, AVI.

KUSKE, A. \& ROBERTSON, G. 1974. Photoelastic stress analysis, Chichester, Wiley Interscience.

ROSENTHAL, A. J. 2015. Instrumental characterisation of solid and semisolid foods. In: CHEN, J. \& ROSENTHAL, A. (eds.) Modifying Food Texture: Sensory Analysis, Consumer Requirements and Preferences. Cambridge, UK: Woodhead Publishing.
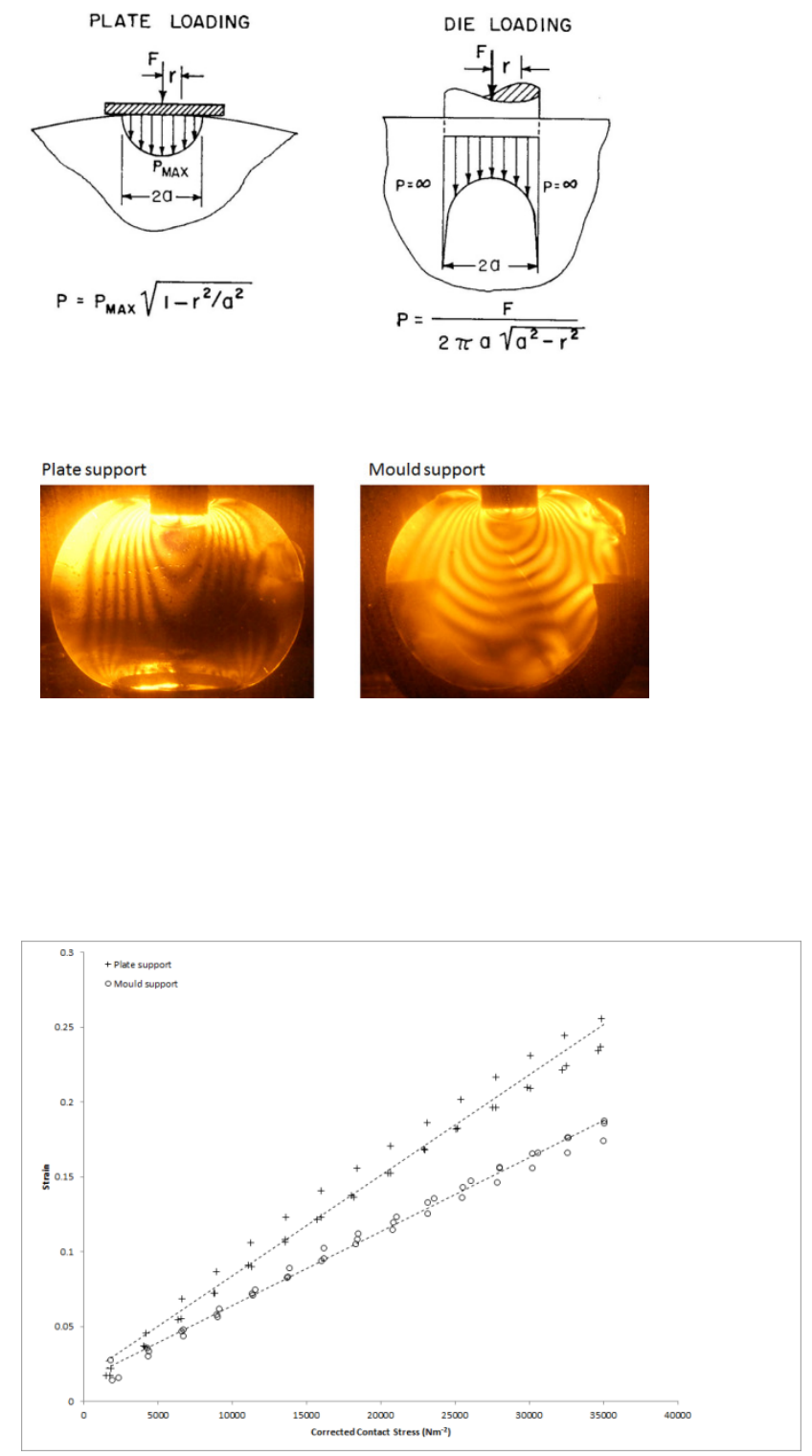